\title{
Julian Mischi, Le Bourg et l'Atelier. Sociologie du combat syndical
}

Marseille, Agone, coll. «L'ordre des choses », 2016

\section{Alexandre Hobeika}

\section{OpenEdition}

\section{Journals}

Édition électronique

URL : http://journals.openedition.org/travailemploi/7550

DOI : $10.4000 /$ travailemploi.7550

ISSN : 1775-416X

Éditeur

DARES - Ministère du Travail

Édition imprimée

Date de publication : 1 avril 2017

Pagination : 138-141

ISSN : 0224-4365

Référence électronique

Alexandre Hobeika, « Julian Mischi, Le Bourg et I'Atelier. Sociologie du combat syndical », Travail et Emploi [En ligne], 150 | avril-juin 2017, mis en ligne le 11 juillet 2019, consulté le 24 septembre 2020. URL

http://journals.openedition.org/travailemploi/7550; DOI : https://doi.org/10.4000/travailemploi.7550 


\section{Le Bourg et l'Atelier. Sociologie du combat syndical}

\section{Julian Mischi}

Marseille, Agone, coll. « L'ordre des choses », 2016, 408 p.

\section{Lu par Alexandre Hobeika*}

Après plusieurs ouvrages consacrés au Parti communiste français (PCF), Julian Mischi se penche sur la Confédération générale du travail (CGT), cousine du parti, qui s'en est éloignée et reste, à sa différence, l'une des principales organisations de représentation des classes populaires. Aujourd'hui l'une des forces politiques les plus étudiées de France, elle fait ici l'objet d'une enquête originale par le terrain, un bourg rural, et la méthode, une ethnographie s'étalant sur une période de cinq ans. L'enquête s'intéresse à la Société nationale des chemins de fer français (SNCF), entreprise qui domine l'activité du bourg grâce à un important atelier de réparation d'équipements de signalisation pour les voies ferrées. La CGT, principal syndicat de la SNCF, y est particulièrement implantée. Cette étude approfondie et localisée permet à l'auteur de questionner les logiques de maintien d'une présence syndicale dans une période difficile et d'éclairer les réseaux politiques et militants « de gauche » dans des territoires souvent pensés comme agricoles et conservateurs, voire comme homogènes et vides de structures politiques.

Pour ce faire, le livre se compose de cinq chapitres : après une présentation de l'établissement et de l'implantation différenciée des syndicats en son sein, la progression de l'ouvrage suit les carrières syndicales en documentant respectivement l'engagement, la socialisation, le travail de représentant et le militantisme extra-syndical.

Les particularités du site d'enquête expliquent le maintien de ce syndicalisme (chapitre 1), l'atelier comptant environ $20 \%$ d'adhérents pour la seule CGT. Ses militants sont des ouvriers à statut ou sont devenus agents de maîtrise après une promotion interne. Ils appartiennent aux fractions les plus stables des classes populaires, même si leur entourage connaît une précarité plus forte, notamment leurs conjointes. L'atelier est parvenu à développer son activité et à disposer d'une autonomie relative dans la SNCF, ce qui a longtemps permis le maintien de la filière locale et technicienne d'accession aux postes d'encadrement, même si la managérialisation de l'entreprise a toutefois fini par s'imposer : les cadres y sont aujourd'hui diplômés du supérieur et peu insérés dans la vie locale.

* Centre Maurice Halbwachs (CMH). 
L'auteur souligne à juste titre l'importance, pour comprendre les engagements syndicaux, du couplage entre la sphère professionnelle et les sociabilités hors-travail (chapitre 2). Celles-ci sont structurées par la corésidence dans les cités cheminotes, ainsi que par la densité des réseaux familiaux dans la commune et ses environs, une particularité de la situation « rurale ». Le degré d'insertion dans le milieu cheminot semble un facteur favorable à l'engagement. La domination masculine est également forte, dans l'atelier comme dans le syndicat. Ce chapitre présente deux traits originaux, à commencer par l'articulation entre carrière syndicale et stratégie résidentielle. L'engagement syndical s'inscrit plus largement dans une logique d'établissement local, combinant la reconnaissance dans le milieu professionnel avec l'accession à la propriété d'un logement et la stabilisation conjugale. Le privé est ici compatible avec l'engagement professionnel et non pas un lieu de repli. Également, l'étude des cheminots issus de familles agricoles permet de montrer finement les clivages internes à ce groupe professionnel. Tout en soulignant leur hétérogénéité sociale, l'auteur note qu'ils présentent un rapport très différent de celui des enfants de cheminots au travail (ils sont souvent exploitants agricoles à titre secondaire), à la hiérarchie usinière (étant davantage dans une logique d'ascension sociale individuelle que de confrontation), et aux réseaux de sociabilité, ce qui s'accompagne de perceptions de l'espace social et d'opinions politiques opposées. Ces deux groupes sont toutefois poreux, peuvent se côtoyer dans les loisirs, et des trajectoires d'intégration au milieu cheminot cégétiste sont possibles.

Dans le chapitre 3, l'auteur explique que la CGT s'attache à inculquer des modes de pensée qui visent à la « politisation » des causes, ici conçue comme montée en généralité et rattachement à des débats nationaux, au respect de la discipline organisationnelle et à la non-appropriation individuelle de ressources collectives. Sous cet angle, cette section syndicale ouvrière apparaît plus proche du « centralisme démocratique » du PCF que d'une « anarchie organisée ${ }^{1} »$. Observer la socialisation militante au niveau de l'entreprise permet également de fournir un éclairage complémentaire aux travaux récents sur la formation syndicale. L'auteur relativise l'importance des dispositifs de formation dans le façonnage des habitus militants et montre que leur apprentissage passe surtout par la pratique. Il souligne aussi la forte inertie idéologique du syndicat, les thématiques féministes et antiracistes étant portées par de jeunes militants mais reléguées au second plan par les dirigeants.

Par son analyse de la position de permanent syndical (chapitre 4), l'auteur peut discuter avec finesse les débats sur la professionnalisation du syndicalisme. Il documente le travail auquel se livrent ces ouvriers militants pour à la fois acquérir des savoirfaire mobilisant des schèmes abstraits et parvenir à une forme de réassurance sociale nécessitée par leur absence de diplôme. Ces compétences placent les permanents dans une situation instable : d'un côté, elles leur confèrent des aspirations à une promotion sociale, de l'autre, ils sont tenus par le groupe de travail qu'ils représentent, d'autant

1. Piotet F. (2009), « La CGT, une anarchie (plus ou moins) organisée ? », Politix, nº 85, pp. 9-30. 
plus fortement que les scènes professionnelles et résidentielles sont superposées. Le retour au travail ouvrier étant subjectivement impossible, les deux seules possibilités de sortie sont un reclassement dans la hiérarchie de l'entreprise ou bien dans celle du syndicat mais, dans les deux cas, au prix de l'éloignement du milieu ouvrier local.

Le dernier chapitre (5) adopte là encore un angle original, celui de l'action militante de ces syndicalistes hors du cadre professionnel. L'auteur montre comment ils tentent de transposer des schèmes d'analyse et des principes d'organisation dans d'autres associations, comme celles de parents d'élèves, tout en mettant en évidence que ces principes peuvent y apparaître désajustés, la pluralité des habitus sociaux et militants auxquels ils doivent se confronter s'accordant mal d'un fonctionnement discipliné et hiérarchique. Il souligne enfin que la socialisation militante à la CGT entretient un intérêt pour la politique, qui ne se traduit pas forcément dans un engagement partisan, du fait de la force des clivages sociaux. Les syndicalistes étudiés, devenus « intellectuels d'institution », semblent en effet en tension avec les « intellectuels de formation ${ }^{2} \gg$ qu'ils rencontrent dans le militantisme de gauche. Plusieurs se déclarent « communistes sans le parti », ayant refusé d'adhérer au PCF durant sa période « stalinienne ». Sur d' autres enjeux plus ciblés, relatifs à la SNCF, des alliances avec les associations d'usagers s'avèrent également impossibles. Avoir acquis des dispositions, des ressources et de l'intérêt pour l'action collective au-delà du cadre professionnel est donc loin de suffire pour nouer des alliances transclassistes.

L'ouvrage de J. Mischi apporte donc de riches éléments sur le rapport au politique des milieux populaires en milieu rural aujourd'hui et sur le syndicalisme dans les grandes entreprises publiques. Le texte est dans l'ensemble bien écrit et sa structure est claire, en dépit de quelques redites.

On peut néanmoins pointer quelques limites, qui ont trait principalement à la stratégie d'enquête, centrée sur le groupe militant CGT et plus particulièrement sur ses dirigeants. Ce choix permet certes à l'auteur d'analyser ces cas de façon subtile; dans le même temps, son point de vue se détache difficilement de celui de ses alliés d'enquête, dont la position centrale dans l'organisation étudiée offre des avantages d'enquête mais n'est pas forcément la plus propice à la construction d'un regard distancié. Les discours critiques à l'égard du syndicat, par des concurrents ou des anciens (début du chapitre 3), sont très éclairants et auraient gagné à être plus nombreux. Le texte aurait également pu mobiliser davantage d'outils d'objectivation, comme des statistiques simples, qui auraient permis de contextualiser les phénomènes décrits. Enfin, l'auteur aurait pu utiliser encore plus le caractère ethnographique de son enquête, par exemple pour décrire les formes de sociabilité hors-travail et entrer plus avant dans les mondes privés.

Mais la principale frustration vient de la construction de l'objet. S'il apprend beaucoup sur les réseaux militants, la socialisation et l'ordre interne à la CGT, le texte

\footnotetext{
2 Pudal B. (1988), «Les dirigeants communistes. Du "fils du peuple” à "l'instituteur des masses" », Actes de la recherche en sciences sociales, $\mathrm{n}^{\circ} 71-72$, pp. 46-70.
} 
n'aborde en revanche que très peu l'action et les enjeux matériels du syndicalisme. Ces derniers apparaissent incidemment mais sont rarement traités de façon approfondie. Sur des questions aussi centrales que les négociations salariales et les conditions de travail, la régulation des carrières, l'usage du comité d'entreprise, l'organisation des grèves, l'auteur nous restitue les discours des syndicalistes mais peu leurs pratiques, si bien que le lecteur peine à estimer les capacités réelles du syndicat à peser. Les différents services aux adhérents sont comme «invisibilisés », peut-être parce que le «syndicalisme de services » est, dans le discours CGT, un terme qui sert à délégitimer les organisations concurrentes. On peut imaginer que l'auteur revendique un positionnement à la fois savant et militant, comme la collection qu'il codirige et dans laquelle le livre est publié. La question de ce qu'on refuse d'objectiver par souci de préservation de l'organisation étudiée est de plus légitime. Mais elle mériterait d'être traitée plus frontalement, ce qui limiterait le risque de donner une vision tronquée du « combat syndical » (sous-titre de l'ouvrage).

En dépit de ces quelques réserves, l'ouvrage de J. Mischi, travail solide et informé, permet de documenter les structures politiques dans les territoires ruraux et leurs dynamiques contemporaines. 
